\title{
Surveillance or metastasis-directed Therapy for OligoMetastatic Prostate cancer recurrence (STOMP): study protocol for a randomized phase II trial
}

Karel Decaestecker ${ }^{1}$, Gert De Meerleer ${ }^{2}$, Filip Ameye ${ }^{3}$, Valerie Fonteyne ${ }^{2}$, Bieke Lambert ${ }^{4}$, Steven Joniau ${ }^{5}$, Louke Delrue ${ }^{6}$, Ignace Billiet ${ }^{7}$, Wim Duthoy ${ }^{8}$, Sarah Junius ${ }^{9}$, Wouter Huysse ${ }^{6}$, Nicolaas Lumen ${ }^{1}$ and Piet Ost ${ }^{2}$

\begin{abstract}
Background: Metastases-directed therapy (MDT) with surgery or stereotactic body radiotherapy (SBRT) is emerging as a new treatment option for prostate cancer (PCa) patients with a limited number of metastases $(\leq 3)$ at recurrence - so called "oligometastases". One of the goals of this approach is to delay the start of palliative androgen deprivation therapy (ADT), with its negative impact on quality of life. However, the lack of a control group, selection bias and the use of adjuvant androgen deprivation therapy prevent strong conclusions from published studies.

The aim of this multicenter randomized phase II trial is to assess the impact of MTD on the start of palliative ADT compared to patients undergoing active surveillance.

Methods/Design: Patients with an oligometastatic recurrence, diagnosed on choline PET/CT after local treatment with curative intent, will be randomised in a 1:1 ratio between arm A: active surveillance only and arm B: MTD followed by active surveillance. Patients will be stratified according to the location of metastasis (node vs. bone metastases) and PSA doubling time ( $\leq 3$ vs. > 3 months). Both surgery and SBRT are allowed as MDT. Active surveillance means 3-monthly PSA testing and re-imaging at PSA progression. The primary endpoint is ADT-free survival. ADT will be started in both arms at time of polymetastatic disease (>3 metastatic lesions), local progression or symptoms. The secondary endpoints include progression-free survival, quality of life, toxicity and prostate-cancer specific survival.
\end{abstract}

Discussion: This is the first randomized phase 2 trial assessing the possibility of deferring palliative ADT with MDT in oligometastatic PCa recurrence.

Trial registration: Clinicaltrials.gov identifier: NCT01558427

Keywords: Oligometastases, Prostate cancer, Salvage treatment, Stereotactic body radiotherapy, Salvage lymph node dissection, Active surveillance, Androgen deprivation therapy, Quality of life, Survival

\section{Background}

Prostate cancer $(\mathrm{PCa})$ is the most frequent tumour in males and $\mathrm{PCa}$ death is attributed to metastatic disease in the majority of patients [1]. The first line treatment of metastatic $\mathrm{PCa}$ is lifelong androgen deprivation therapy (ADT) by means of surgical or medical castration [1]. Although this strategy delays PCa progression, it is associated with numerous side effects impacting quality-of-

\footnotetext{
* Correspondence: piet.ost@ugent.be

${ }^{2}$ Department of Radiotherapy, Ghent University Hospital, Ghent, Belgium Full list of author information is available at the end of the article
}

life and general health $[2,3]$. There is no proven overall survival benefit of immediate ADT over deferred ADT in metastatic patients [1]. It is therefore an option in the EAU guidelines to suggest an active surveillance protocol for well-informed asymptomatic patients with PCA metastases [1].

In analogy with other solid tumours, eradication of these oligometastases by means of metastases directed therapy (MDT) with stereotactic body radiotherapy (SBRT) or surgery is a promising and emerging way to delay disease progression and postpone systemic treatment without major 
treatment toxicity [4]. However, two major difficulties complicate assessment of the benefit of radical treatment for oligometastastatic prostate cancer recurrence.

Firstly, the identification of patients with oligometastatic disease is challenging. The traditional imaging studies such as bone scan and computed tomography lack sufficient sensitivity to detect low volume metastatic disease at low PSA levels [5]. Consequently, these imaging modalities are not recommended to screen patients for metastases until the PSA reaches $10 \mathrm{ng} / \mathrm{ml}^{19}$. Choline positron emitting tomography (PET) with computed tomography (CT) might be a good candidate for the identification of low volume metastases with a pooled sensitivity and specificity exceeding $85 \%$ on a per-patient basis in the recurrent setting $[6,7]$.

Secondly, the apparent achievement of MDT for oligometastatic $\mathrm{PCa}$ in postponing palliative $\mathrm{ADT}$ and in slowing down progression has only been observed in single arm studies $[8,9]$. The true benefit of this MDT can only be assessed through randomization versus a control group. Otherwise, it could be that the promising results are only due to selection of fit patients with very slow-growing tumors, rather than the result of treatment intervention itself.

The current study will address these shortcomings by randomizing MDT versus active surveillance. The active surveillance arm will inform us about the natural progression of oligometastatic PCa.

This study is the first randomized study in this setting and will employ a randomized phase II design to determine which arm is justified to be tested in a subsequent phase III trial.

\section{Methods/design}

This study is approved by the Ethics committee of the Ghent University Hospital (EC2012/156) and is registered on clinicaltrials.gov (NCT01558427). The protocol is designed according to the clinical-state model [10]: rising PSA and clinical metastases state in non-castrate patients. Patients diagnosed with oligometastases (up to $3 \mathrm{~N} 1$ or M1a/b lesions [1]) will be entered in a randomized Phase II trial. In the experimental arm A, patients will undergo active clinical surveillance with the start of $\mathrm{ADT}$ at progression. In arm B, patients will receive SBRT or surgery of the oligometastases, followed by active clinical surveillance.

\section{Objectives}

- Primary endpoint

$\circ$ Androgen deprivation therapy free survival ADT will be started in both arms at time of polymetastatic disease, local progression (defined below) or symptoms. In case of a metachronous oligometastatic recurrence in arm A, a retreatment with radiotherapy or surgery is allowed. Calculation will start from randomization until ADT is started.

- Secondary endpoints

$\circ$ Quality of life scoring using the EORTC QLQ-C30 supplemented with QLQ-PR25. Raw scores will be transformed to a linear scale ranging from 0 to 100 . The results will be presented in accordance with recent guidelines for reporting HRQOL RCTs [11].

- Assessment of quality-adjusted-life-years with the EuroQol classification system (EQ-5D) [12]. A written consent to use this system has been obtained from the EuroQol Group Foundation.

- Acute and late toxicity due to radiotherapy will be scored using the Common toxicity criteria version 4.0 [13]. Surgical complications will be scored using Clavien-Dindo Classification [14]. Other surgical related morbidity (intra-operative complications (blood loss, injury to pelvic or intra-abdominal organs...) and duration of hospital stay) will also be recorded.

- Time to castration-resistant disease is calculated from randomization until development of castration-resistant disease as defined by the EAU guidelines [1].

- Progression-free survival:

- 3 types of progression are defined and definitions of progression are used and registered according to the recommendations of the prostate cancer clinical trials working group [10]. Calculation will start from randomization until progression or death.

- PSA or biochemical progression:

- In case of decline from baseline: record time from randomization to first PSA increase that is $\geq 25 \%$ and $\geq 2 \mathrm{ng} / \mathrm{ml}$ above the nadir OR that is $\geq 25 \%$ and rises above the pretreatment PSA value and which is confirmed by a second value 3 or more weeks later.

- In case of no decline from baseline: PSA increase that is $\geq 25 \%$ and $\geq 2 \mathrm{ng} / \mathrm{ml}$ after 3 months if baseline PSA is $\geq 2 \mathrm{ng} / \mathrm{ml}$. PSA increase that is $\geq 25 \%$ after 3 months if baseline PSA is $<2 \mathrm{ng} / \mathrm{ml}$.

- Local progression for soft-tissue and bone lesions: Each metastasis is a target lesion independently assessed for response with the RECIST criteria [15]. In addition, metastases (particularly osseous) with a metabolic complete response on bone or PET scan are scored as complete response in the absence of progression on CT scan. 
- Distant progression: appearance of new metastatic lesions.

- Prostate cancer specific survival will be calculated from randomization until $\mathrm{PCa}$ death. Overall survival will be calculated from randomization until death from any cause.

- Time to first symptomatic event will be calculated from randomization until the event of symptoms due to metastatic disease.

\section{Inclusion criteria}

- Histologically proven diagnosis of PCa

- PCa patients with a biochemical recurrence following treatment with curative intent (radical prostatectomy, primary radiotherapy or a combination of both) as defined by the EAU guidelines [1].

- A maximum of 3 extracranial metastases in any organ system diagnosed on choline PET-CT.

- Controlled primary tumor

o Patients in the postoperative setting should have received postoperative radiotherapy to the prostate bed. In case the PSA $>2 \mathrm{ng} / \mathrm{ml}$ in the postoperative setting patients are eligible if a multiparametric MRI of the prostate bed rules out a local relapse or a negative biopsy of the prostate bed is performed ${ }^{37}$. Patients after primary radiotherapy should undergo MRI of the prostate according to the European Society of Urogenital Radiology (ESUR) guidelines to rule out local relapse [16]. In case of a suspicious lesion, a biopsy should confirm local recurrence and patients should be referred for local salvage prostatectomy when distant metastases are ruled out. If MRI rules out local relapse, patients are eligible.

- WHO performance state 0-1

- Age $\geq 18$ years old

- Willing to provide a signed informed consent

- Patient presented at the multidisciplinary tumour board of the local hospital in which the therapy will be given.

\section{Exclusion criteria}

- Serum testosterone level $<50 \mathrm{ng} / \mathrm{ml}$

- Symptomatic metastases

- Patients with oligometastases that have been previously treated.

- PSA rise while on active treatment (LHRH-agonist, LHRH-antagonist, anti-androgen, maximal androgen blockade, oestrogen)

- Previous treatment with a cytotoxic agent for PCa
- Treatment during the past month with products known to influence PSA levels (e.g. fluconazole, finasteride, corticosteroids,...)

- Disorder precluding understanding of trial information or informed consent

\section{Evaluation and randomization}

Patients must be restaged within 4 weeks prior to randomization with $18 \mathrm{~F}$ or $11 \mathrm{C}$-choline PET-CT. MRI full spine and bony pelvis or MRI whole body is optional, but recommended in case of equivocal findings. Bone scintigraphy is not required.

The study will employ a 1:1 randomization between $\operatorname{arm~A:~arm~B.~Patients~will~be~stratified~according~to~}$ PSA doubling time and ( $\leq 3$ vs. $>3$ months) and initial localization of metastases (node vs bone or visceral).

\section{Interventions}

1. Arm A: Active clinical surveillance

- Defined as 3-monthly clinical examination and serum PSA measurement. Restaging will be performed in case of symptomatic progression or PSA progression:

- A PSA increase that is $\geq 25 \%$ and $\geq 2 \mathrm{ng} / \mathrm{ml}$ if baseline PSA is $\geq 2 \mathrm{ng} / \mathrm{ml}$.

- A PSA increase that is $\geq 25 \%$ if baseline PSA is $<2 \mathrm{ng} / \mathrm{ml}$.

- ADT will be started at time of polymetastatic disease, local progression (defined above) or symptoms. The type of ADT is left to the discretion of the treating physician. Both anti-androgen monotherapy, LHRH agonist or antagonists and maximal androgen deprivation therapy are allowed. Both intermittent and continuous ADT are allowed.

\section{Arm B}

a. SBRT and surgery.

All patients randomized into arm A will be presented at the multidisciplinary urology tumour board prior to treatment. The choice between SBRT or surgery will depend on localization and size of the metastases, the nearby organs-at-risk and previous treatments in the vicinity of the metastases. After reaching a consensus, the patient will be informed about the options for treatment.

b. SBRT target and organ-at-risk definition. All patients will receive a $\mathrm{CT}$ simulation in supine position with $2 \mathrm{~mm}$ CT slice thickness through the tumour site. The planning simulation should cover the target and all organs at risk. A typical scan length 
should extend at least $10 \mathrm{~cm}$ superior and inferior beyond the treatment field borders. Support devices to increase patient comfort will be chosen depending on the tumour localisation. The isocenter will be determined on the CT-simulator with marking of laser lines on the patient. Imaging data will be transferred to the treatment planning system. For all lesions, the Gross Target Volume (GTV) will be defined as all visible tumor by combining iconographic and metabolic information. No additional margin will be added for microscopic spread of disease. The GTV will be expanded with 2-5 $\mathrm{mm}$ to the Planning Target Volume (PTV) to account for organ motion and setup error. Margins depend on the site irradiated with $2 \mathrm{~mm}$ margins for bony lesions, $3 \mathrm{~mm}$ for nodes and $5 \mathrm{~mm}$ for other sites. The type of organ at risk delineated depends on the localization of the metastasis. A Planning Organ at Risk Volume (PRV) expansion of 2-5 mm will be added for OAR such as the spinal cord, oesophagus, intestine,... (if applicable), and dose constraints apply to this PRV. It is strongly recommended that dose constraints not be exceeded. If a dose constraint cannot be achieved due to overlap of the target with an organ at risk or its PRV, the total dose can be lowered in order to meet the constraint.

For spinal lesions, a pre-treatment axial MRI is required to assess the extent of disease and position of the cord. This must be fused with the planning CT scan.

c. Radiotherapy treatment planning and dose prescription:

IMRT (static or rotational) treatment planning will be dependent the localization of the metastasis. Dose constraints for organ at risks will be in accordance with the recommendations from the report of the AAPM task group 101 [17]. A total dose of 30 Gy ( $80 \%$ of the maximal dose) will be delivered in 3 fractions and fractions will be separated $>48 \mathrm{~h}$ and $<96 \mathrm{~h}[18,19]$.

Treatment will be prescribed to the periphery of the target ( $80 \%$ of the dose (=30 Gy), should cover $90 \%$ of the PTV). In case of violation of dose constraints to the organs at risk, the prescription will be adapted accordingly. If multiple targets will be irradiated and the targets are more than $10 \mathrm{~cm}$ apart in the cranio-caudal direction, multiple isocenters are needed with a CBCT prior to every treatment for every isocenter. Patient immobilization devices can be used according to the institutional policy.

d. Radiotherapy delivery and verification: In order to ensure patient safety and effective treatment delivery the following measurements are taken:

- Prior to treatment, all plans are discussed and approved at the daily radiotherapy rounds after prior verification by the treating physician.

- All dose delivery for intensity-modulated plans will be confirmed before treatment by physics staff.

- At each fraction, a cone-beam CT (CBCT) will be used for patients' set-up and target verification prior to treatment. In case of multiple isocenters, every isocenter will be verified separately with CBCT.

- Quality assurance: all plans will be verified using the Delta(4) diode array phantom prior to treatment delivery [20].

e. Surgical procedures:

The surgical technique to be used is at the discretion and expertise of the surgeon but must be in accordance to the best surgical practice available. A minimally invasive technique is preferred, but not obligatory. For pelvic nodal metastases, in case of a previous extended pelvic lymph node dissection (ePLND) or pelvic radiotherapy, only the suspicious lymph node will be removed. If no ePLND has been performed previously, a salvage ePLND will be preferred. In case of retroperitoneal nodes, only the suspicious node will be removed.

f. Androgen deprivation therapy:

ADT will be started as in arm A. In case of a metachronous oligometastatic recurrence, a retreatment with SBRT or surgery is allowed.

\section{Follow-up}

Patients will be seen every 3 months post-randomization (Table 1). At each visit, a history and physical examination will conducted with recording of the toxicity. The QOL questionnaires will be concluded at each visit. Choline PET-CT will be repeated at PSA or symptomatic progression. Additional Imaging or laboratory investigations should be carried out at the discretion of the treating physician, based on findings in the history or physical, and additional treatment (e.g. ADT). 
Table 1 Study visits and procedures

\begin{tabular}{llll}
\hline & At inclusion & 3-monthly & $\begin{array}{l}\text { At PSA or } \\
\text { symptomatic } \\
\text { progression }\end{array}$ \\
\hline Eligibility check & $*$ & & \\
Informed consent & $*$ & & $*$ \\
PET-CT & $*$ & $*$ & $*$ \\
QOL questionnaire & $*$ & $*$ & $*$ \\
Toxicity assessment & $*$ & $*$ & $*$ \\
PSA measurement & $*$ & $*$ & $*$ \\
History and physical & $*$ & & \\
examination & & & \\
\hline
\end{tabular}

${ }^{*}$ reflects the timepoint of a certain assessment.

\section{Statistical analysis Sample size}

This study will employ a randomized phase II design to determine which arm is justified to be tested in a subsequent phase III trial. The study will therefore be designed with an alpha and beta $=0.20$ (as recommended for phase II randomized trials [21,22]). It is estimated that the median delay to start palliative ADT after metastasis-directed therapy is approximately 24 months [4]. There will be a 1:1 randomization between Arm 1 and Arm 2. Patients will be stratified according to PSA doubling time ( $\leq 3$ vs. $>3$ months) and initial localization of metastases (node vs bone or visceral). In order to detect a 12-month difference in the studied endpoint from 12 to 24 months, a total of 58 patients will be needed. Assuming a 5\% rate of loss to follow-up, a total of 62 patients will be accrued over 36 months with 12 months of additional follow-up. We expect an accrual rate of 20 patients per year.

\section{Data analysis}

Patients will be analysed in the groups to which they are assigned (intention-to-treat). The primary endpoint androgen deprivation therapy-free survival will be calculated using Kaplan-Meier actuarial analyses. Pre-planned subgroup analysis will occur based on stratification variables using the log-rank test. Survival times are defined from the day of randomization until an event (start of ADT) or last follow-up. Cases will be censored at last follow up visit if no ADT was started. Multivariate analysis will be performed according to the Cox-Regression method.

Overall survival and progression-free survival (local, biochemical and clinical progression free survival) will be evaluated in the same way as the primary endpoint. All $\mathrm{p}$ values are set at 0.05 . Statistical analysis will be performed with SPSS (IBM Corp, Somers, NY, USA).

\section{Discussion}

The standard treatment options for PCa patients diagnosed with metastatic progression following primary treatment have remained unchanged over the past years [23], with ADT being the cornerstone of treatment [1]. Several small, single arm observational studies have been reported with promising results $[4,8,9,19,24-26]$. However the rather random use of a multimodality approach with adjuvant ADT and prophylactic nodal irradiation in these studies makes it difficult to make any definite conclusions. This is the first randomized phase 2 trial that will asses the possibility of deferring palliative ADT and cancer progression with MDT by means of SBRT or surgery. The inclusion of an active surveillance arm will improve our insights in the natural progression of oligometastatic PCa.

\section{Abbreviations \\ PCa: Prostate cancer; ADT: Androgen deprivation therapy; EAU: European Association of Urology; PET-CT: Positron emission tomography; \\ $\mathrm{CT}$ : Computed tomography; MRI: Magnetic resonance imaging; AS-MRI: Axial skeleton MRI; WB-MRI: Whole body MRI; PSA: Prostate specific antigen; SBRT: Stereotactic body radiotherapy; ePLND: Extended pelvic lymph node dissection; IMRT: Intensity modulated radiotherapy; AAPM: American Association of Physicists in Medicine; GTV: Gross target volume; PTV: Planned target volume; PRV: Planning organs at risk volume; RT: Radiotherapy; QOL: Quality of life; EORTC: European Organization for Research and Treatment of Cancer; RTOG: Radiation Therapy Oncology Group; WHO: World Health Organization; ESUR: European Society of Urogenital Radiology; LHRH: Luteinizing hormone releasing hormone.}

\section{Competing interests}

The authors declare that they have no competing interests.

\section{Author's contributions}

Study conception: GD, PO. Initial Study design: PO, GD, KD, NL, GV, BL, Revision of study design and protocol: PO, GD, KD, NL, LD, VF, GV, WH, BL. Study coordination: PO, KD. Participating centers: WD, FA, IB, SaJ, StJ, PS. Drafting the manuscript: all authors. All authors read and approved the final manuscript.

\section{Acknowledgements}

This study is funded by a grant of the Vlaamse Liga tegen Kanker (VLK). The granting bodies are not involved in data collection or analysis. Dr. Piet Ost is a senior Clinical Investigator of the Research Foundation - Flanders (Belgium) (FWO).

\section{Author details}

${ }^{1}$ Department of Urology, Ghent University Hospital, Ghent, Belgium. ${ }^{2}$ Department of Radiotherapy, Ghent University Hospital, Ghent, Belgium. ${ }^{3}$ Department of Urology, AZ Maria Middelares Gent, Ghent, Belgium. ${ }^{4}$ Department of Nuclear Medicine, Ghent University Hospital, Ghent, Belgium. ${ }^{5}$ Department of Urology, University Hospitals Leuven, Leuven, Belgium. ${ }^{6}$ Department of Radiology, Ghent University Hospital, Ghent, Belgium. 7 Department of Urology, AZ Groeninghe, Kortrijk, Belgium. ${ }^{8}$ Department of Radiotherapy, AZ Sint-Lucas Gent, Ghent, Belgium. ${ }^{9}$ Department of Radiotherapy, CH Mouscron, Mouscron, Belgium.

Received: 6 June 2014 Accepted: 2 September 2014

Published: 15 September 2014

\section{References}

1. Heidenreich A, Bastian PJ, Bellmunt J, Bolla M, Joniau S, van der Kwast T, Mason M, Matveev V, Wiegel T, Zattoni F, Mottet N: EAU guidelines on prostate cancer. part II: treatment of advanced, relapsing, and castration-resistant prostate cancer. Eur Urol 2014, 65(2):467-479. 
2. Abrahamsson PA: Potential benefits of intermittent androgen suppression therapy in the treatment of prostate cancer: a systematic review of the literature. Eur Urol 2010, 57(1):49-59.

3. Taylor LG, Canfield SE, Du XL: Review of major adverse effects of androgen-deprivation therapy in men with prostate cancer. Cancer 2009, 115(11):2388-2399.

4. Decaestecker K, De Meerleer G, Lambert B, Delrue L, Fonteyne V, Claeys T, De Vos F, Huysse W, Ost P: Repeated stereotactic body radiotherapy for oligometastatic prostate cancer recurrence. Radiat Oncol 2014, in press.

5. Mottet N, Bellmunt J, Bolla M, Joniau S, Mason M, Matveev V, Schmid HP, Van der Kwast T, Wiegel T, Zattoni F, Heidenreich A: EAU guidelines on prostate cancer. part II: treatment of advanced, relapsing, and castration-resistant prostate cancer. Eur Urol 2011, 59(4):572-583.

6. Umbehr MH, Muntener M, Hany T, Sulser T, Bachmann LM: The role of 11C-choline and 18 F-fluorocholine positron emission tomography (PET) and PET/CT in prostate cancer: a systematic review and meta-analysis. Eur Urol 2013, 64(1):106-117.

7. Wondergem $M$, van der Zant FM, van der Ploeg T, Knol RJ: A literature review of 18 F-fluoride PET/CT and 18 F-choline or 11C-choline PET/CT for detection of bone metastases in patients with prostate cancer. Nucl Med Commun 2013, 34(10):935-945.

8. Berkovic P, De Meerleer G, Delrue L, Lambert B, Fonteyne V, Lumen N, Decaestecker K, Villeirs G, Vuye P, Ost P: Salvage stereotactic body radiotherapy for patients with limited prostate cancer metastases: deferring androgen deprivation therapy. Clin Genitourin Cancer 2013, 11(1):27-32

9. Suardi N, Gandaglia G, Gallina A, Di Trapani E, Scattoni V, Vizziello D, Cucchiara V, Bertini R, Colombo R, Picchio M, Giovacchini G, Montorsi F, Briganti A: Long-term outcomes of salvage lymph node dissection for clinically recurrent prostate cancer: results of a single-institution series with a minimum follow-up of 5 years. Eur Urol 2014, doi:10.1016/j.eururo.2014.02.011

10. Scher HI, Halabi S, Tannock I, Morris M, Sternberg CN, Carducci MA, Eisenberger MA, Higano C, Bubley GJ, Dreicer R, Petrylak D, Kantoff P, Basch E, Kelly WK, Figg WD, Small EJ, Beer TM, Wilding G, Martin A, Hussain M, Prostate Cancer Clinical Trials Working Group: Design and end points of clinical trials for patients with progressive prostate cancer and castrate levels of testosterone: recommendations of the Prostate Cancer Clinical Trials Working Group. J Clin Oncol 2008, 26(7):1148-1159.

11. Bottomley A, Flechtner $H$, Efficace $F$, Vanvoorden V, Coens $C$, Therasse $P$, Velikova G, Blazeby J, Greimel E, European Organisation for Research and Treatment of Cancer: Health related quality of life outcomes in cancer clinical trials. Eur J Cancer 2005, 41(12):1697-1709.

12. EuroQol Group: EuroQol-a new facility for the measurement of health-related quality of life. the EuroQol Group. Health Policy 1990, 16(3):199-208

13. Trotti $A$, Colevas $A D$, Setser $A$, Rusch $V$, Jaques $D$, Budach $V$, Langer $C$, Murphy B, Cumberlin R, Coleman CN, Rubin P: CTCAE v3.0: development of a comprehensive grading system for the adverse effects of cancer treatment. Semin Radiat Oncol 2003, 13(3):176-181.

14. Mitropoulos D, Artibani W, Graefen M, Remzi M, Roupret M, Truss M: Reporting and grading of complications after urologic surgical procedures: an ad hoc EAU guidelines panel assessment and recommendations. Eur Urol 2012, 61(2):341-349.

15. Eisenhauer EA, Therasse P, Bogaerts J, Schwartz LH, Sargent D, Ford R, Dancey J, Arbuck S, Gwyther S, Mooney M, Rubinstein L, Shankar L, Dodd L, Kaplan R, Lacombe D, Verweij J: New response evaluation criteria in solid tumours: revised RECIST guideline (version 1.1). Eur J Cancer 2009, 45(2):228-247.

16. Barentsz JO, Richenberg J, Clements R, Choyke P, Verma S, Villeirs G, Rouviere O, Logager V, Futterer JJ, European Society of Urogenital Radiology: ESUR prostate MR guidelines 2012. Eur Radiol 2012, 22(4):746-757

17. Benedict SH, Yenice KM, Followill D, Galvin JM, Hinson W, Kavanagh B, Keall P, Lovelock M, Meeks S, Papiez L, Purdie T, Sadagopan R, Schell MC, Salter B, Schlesinger DJ, Shiu AS, Solberg T, Song DY, Stieber V, Timmerman R, Tome WA, Verellen D, Wang L, Yin FF: Stereotactic body radiation therapy: the report of AAPM Task Group 101. Med Phys 2010, 37(8):4078-4101.

18. Salama JK, Hasselle MD, Chmura SJ, Malik R, Mehta N, Yenice KM, Villaflor VM, Stadler WM, Hoffman PC, Cohen EE, Connell PP, Haraf DJ, Vokes EE, Hellman S, Weichselbaum RR: Stereotactic body radiotherapy for multisite extracranial oligometastases: final report of a dose escalation trial in patients with 1 to 5 sites of metastatic disease. Cancer 2012, 118(11):2962-2970.

19. Casamassima F, Masi L, Menichelli C, Bonucci I, Casamassima E, Lazzeri M, Gulisano M, Aterini S: Efficacy of eradicative radiotherapy for limited nodal metastases detected with choline PET scan in prostate cancer patients. Tumori 2011, 97(1):49-55.

20. Bedford JL, Lee YK, Wai P, South CP, Warrington AP: Evaluation of the Delta4 phantom for IMRT and VMAT verification. Phys Med Biol 2009, 54(9):N167-N176.

21. Rubinstein LV, Korn EL, Freidlin B, Hunsberger S, Ivy SP, Smith MA: Design issues of randomized phase II trials and a proposal for phase II screening trials. J Clin Oncol 2005, 23(28):7199-7206.

22. Palma DA, Haasbeek CJ, Rodrigues GB, Dahele M, Lock M, Yaremko B, Olson R, Liu M, Panarotto J, Griffioen GH, Gaede S, Slotman B, Senan S: Stereotactic ablative radiotherapy for comprehensive treatment of oligometastatic tumors (SABR-COMET): study protocol for a randomized phase II trial. BMC Cancer 2012, 12:305.

23. Palmbos PL, Hussain M: Non-castrate metastatic prostate cancer: have the treatment options changed? Semin Oncol 2013, 40(3):337-346.

24. Jilg CA, Rischke HC, Reske SN, Henne K, Grosu AL, Weber W, Drendel V, Schwardt M, Jandausch A, Schultze-Seemann W: Salvage lymph node dissection with adjuvant radiotherapy for nodal recurrence of prostate cancer. J Urol 2012, 188(6):2190-2197.

25. Schick U, Jorcano S, Nouet P, Rouzaud M, Vees H, Zilli T, Ratib O, Weber DC, Miralbell R: Androgen deprivation and high-dose radiotherapy for oligometastatic prostate cancer patients with less than five regional and/or distant metastases. Acta Oncol 2013, 52(8):1622-1628.

26. Jereczek-Fossa BA, Beltramo G, Fariselli L, Fodor C, Santoro L, Vavassori A, Zerini D, Gherardi F, Ascione C, Bossi-Zanetti I, Mauro R, Bregantin A, Bianchi LC, De Cobelli O, Orecchia R: Robotic image-guided stereotactic radiotherapy, for isolated recurrent primary, lymph node or metastatic prostate cancer. Int $\mathrm{J}$ Radiat Oncol Biol Phys 2012, 82(2):889-897.

\section{doi:10.1186/1471-2407-14-671}

Cite this article as: Decaestecker et al: Surveillance or metastasisdirected Therapy for OligoMetastatic Prostate cancer recurrence (STOMP): study protocol for a randomized phase II trial. BMC Cancer 2014 14:671.

\section{Submit your next manuscript to BioMed Central and take full advantage of:}

- Convenient online submission

- Thorough peer review

- No space constraints or color figure charges

- Immediate publication on acceptance

- Inclusion in PubMed, CAS, Scopus and Google Scholar

- Research which is freely available for redistribution

Submit your manuscript at www.biomedcentral.com/submit
C Biomed Central 